\title{
The evaluation of hepatic fibrosis in patients with chronic hepatitis C, using the FibroMax and FibroScan techniques
}

\author{
Iulia Anca Marian (Deaconu)1, Gheorghe Iulian Diaconescu ${ }^{1,2}$, Valentin Cirlig ${ }^{3}$ \\ ${ }^{1}$ The Infectious Diseases Hospital "Victor Babes", Craiova, Romania \\ ${ }^{2}$ Infectious Diseases Department, University of Medicine and Pharmacy, Craiova, Romania \\ ${ }^{3}$ Pharmacology Department, University of Medicine and Pharmacy, Craiova, Romania
}

\begin{abstract}
This article represents an insight on the latest techniques used for determining the degree of hepatic fibrosis in patients with chronic hepatitis $\mathrm{C}$ - the FibroMax and FibroScan techniques, by not only evaluating their results, but also by comparing and contrasting them. We studied 16 patients, diagnosed and treated in the Infectious Diseases Hospital "Victor Babes" from Craiova, with different degrees of fibrosis (F2-F4) and of viral inflammation (A0-A1-A3). The FibroScan results varied between 7.8 and $66.4 \mathrm{kPa}$. Also, 11 from the 16 patients showed a correspondence between the FibroTest $(F)$ of the FibroMax determination result and the one obtained at FibroScan. Thus, the two methods, although each of them is extremely important, are recommended to be used together for a more accurate quantification of the hepatic fibrosis degree in patients with chronic hepatitis $\mathrm{C}$.
\end{abstract}

Keywords: hepatitis, fibroMax, fibroScan, fibrosis

\section{INTRODUCTION}

Hepatitis $\mathrm{C}$ is an infectious disease which affects the liver and is caused by the hepatitis $\mathrm{C}$ virus (HCV). This virus was discovered during the $80 \mathrm{~s}$ in USA, by the researchers from the Department of Transfusions of the National Health Institute, who noted the emergence of a new hepatitis virus which didn't resemble the A virus, neither the $\mathrm{B}$ virus. This new specimen settled in the hepatic cell determining at first a parenchymal inflammation and afterwards fibrosis and necrosis of the tissue (1).

The evaluation of the degree of hepatic fibrosis can be made using three main techniques: the punch biopsy, using the biological markers (FibroMax technique) and the FibroScan evaluation. The punch biopsy represents an invasive method through which one can obtain hepatic tissue samples in order to submit them to histological evaluation. Even if until a few years ago this procedure was mandatory in diagnosing the degree of hepatic damage induced by the $\mathrm{B}$ and $\mathrm{C}$ virus, today it can be successfully replaced by non-invasive techniques (such as FibroMax and FibroScan), thus reducing the bleeding risks, the pain and even the risk of death by respiratory arrest $(5,7,8)$.

\section{MATERIALS AND METHODS}

In this article we will evaluate the results of 16 patients diagnosed with chronic hepatitis $\mathrm{C}$ admitted and treated in the Adults Clinic II of the Infectious Diseases Hospital "Victor Babes" from Craiova. All the members of this batch performed not only the biological markers analysis FibroMax but also the FibroScan evaluation.

The FibroMax investigation consists of a combination of 5 non-invasive tests - FibroTest, ActiTest, SteatoTest, NashTest and AshTest - and it is based on an algorithm which combines the results 
from some biological markers' determination (alpha-2 macroglobulin, apolipoprotein a1, haptoglobin, gamma glutamyl transpeptidase, total bilirubin, hepatic transaminases - ALAT, ASAT, cholesterol, triglycerides and glycemia) with age, sex, height and weight of every patient, in order to determine the degree of hepatic injury (2).

The FibroTest or the index F of FibroMax measures the degree of hepatic fibrosis (corresponding to the F0-F4 stages from the METAVIR score), ActiTest (A) measures the inflammation degree in chronic hepatitis $\mathrm{B}$ or $\mathrm{C}$ (corresponding to the A0A3 stages of the METAVIR score); SteatoTest evaluates the hepatic steatosis (corresponding to the $0-3$ stages of steatosis: S0-S3); NashTest evaluates the non-alcoholic steatosis in patients with dyslipidemia, obesity, diabetes or insulin resistance (corresponding to the three degrees of the Kleiner classification: N0- "not NASH", N1- "Borderline NASH" and N2-"NASH"); AshTest measures the degree of hepatic injury in patients who are chronic alcohol consumers (corresponding to the H0-H3 degrees) (2).

The FibroScan is an ultramodern non-invasive device which determines the fibrosis degree and also the level of hepatic injury. It quantifies the hepatic fibrosis by analysing the trajectory of an elastic shock wave which propagates through the hepatic tissue (3). The result is the median of ten valid measurements and its unit of measurement is the $\mathrm{kPa}$ (ranging between 2.5 and $75 \mathrm{kPa}$ ). The results' interpretation is: $<7.1 \mathrm{kPa}-$ absent/mild fibrosis ( $\mathrm{f0}$ $\mathrm{F} 1$ ); between 7.1 and $9.5 \mathrm{kPa}-\mathrm{F} 2$ fibrosis; between 9.5 and $13 \mathrm{kPa}-\mathrm{F} 3$ fibrosis; the values which go over $13 \mathrm{kPa}$ mean an F4 fibrosis (hepatic cirrhosis) (4). This test can be made after a doctor's recommendation or by the patient's own will, but, in order to establish a correct diagnosis it is recommended to associate the analysis of the FibroMax markers.

Thus, we made a linear correlation between the FibroMax results of the 16 patients from our batch and their FibroScan evaluations. We also correlated the FibroTest (F) results with the ones from FibroScan.

\section{RESULTS AND DISCUSSSIONS}

By analysing the results of the FibroMax measurements we noticed that 10 out of the 16 studied patients got results which placed them in the F4 stage of FibroTest (the hepatic cirrhosis stage), while only 5 patients got an F3 score and only one an F2.

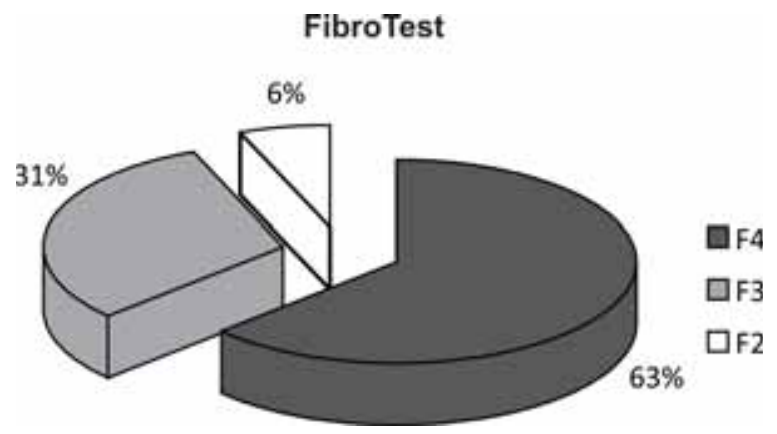

FIGURE 1. Stages of disease using FibroTest

TABLE 1. The results of the FibroMax and FibroScan measurements belongin to the patients from the studied batch

\begin{tabular}{|l|c|c|c|c|c|c|c|c|c|c|c|}
\hline \multirow{2}{*}{$\begin{array}{l}\text { Nr. } \\
\text { Crt. }\end{array}$} & \multicolumn{2}{|c|}{ FibroTest (F) } & \multicolumn{2}{|c|}{ ActiTest (A) } & \multicolumn{2}{c|}{ SteatoTest (S) } & \multicolumn{2}{c|}{ NashTest (N) } & \multicolumn{2}{c|}{ AshTest (H) } & \multicolumn{1}{c|}{ Fibroscan } \\
\hline 1 & Degree & Value & Degree & Value & Degree & Valuee & Degree & Value & Degree & Value & Value(kPa) \\
\hline 2 & 4 & 0.95 & 3 & 0.83 & 1 & 0.4 & 2 & 0.75 & 1 & 0.32 & 31.6 \\
\hline 3 & 4 & 0.85 & 3 & 0.65 & 2 & 0.63 & 1 & 0.5 & 0 & 0.17 & 7.8 \\
\hline 4 & 4 & 0.54 & 3 & 0.62 & $0-1$ & 0.34 & 0 & 0.25 & 0 & 0.04 & 15.4 \\
\hline 5 & 4 & 0.78 & 3 & 0.81 & 2 & 0.62 & 2 & 0.75 & 0 & 0.07 & 20 \\
\hline 6 & 4 & 0.94 & 2 & 0.53 & 0 & 0.28 & 0 & 0.25 & 0 & 0.05 & 15.1 \\
\hline 7 & 3 & 0.6 & $0-1$ & 0.29 & 0 & 0.15 & 0 & 0.25 & 0 & 0.01 & 11.7 \\
\hline 8 & 4 & 0.82 & 3 & 0.76 & s1-s2 & 0.56 & 0 & 0.25 & 0 & 0.06 & 10.5 \\
\hline 9 & 4 & 0.8 & a1-a2 & 0.46 & s1-s2 & 0.52 & 2 & 0.75 & 0 & 0 & 13.6 \\
\hline 10 & 3 & 0.63 & 3 & 0.67 & 3 & 0.77 & 1 & 0.6 & 0 & 0.001 & 12.6 \\
\hline 11 & 4 & 0.88 & 2 & 0.55 & $0-1$ & 0.33 & 0 & 0.25 & 0 & 0.007 & 12.4 \\
\hline 12 & 3 & 0.66 & a1-a2 & 0.47 & 0 & 0.59 & 2 & 0.75 & 0 & 0.002 & 9.9 \\
\hline 13 & 4 & 0.81 & 3 & 0.74 & s1-s2 & 0.49 & 1 & 0.5 & 0 & 0.009 & 66.4 \\
\hline 14 & 3 & 0.61 & a1-a2 & 0.43 & 0 & 0.25 & 0 & 0.25 & 0 & 0.002 & 14.3 \\
\hline 15 & 4 & 0.77 & 3 & 0.62 & s1-s2 & 0.57 & 2 & 0.75 & 0 & 0.11 & 10.1 \\
\hline 16 & 3 & 0.63 & a1-a2 & 0.43 & 0 & 0.23 & 0 & 0.25 & 0 & 0.001 & 10.8 \\
\hline
\end{tabular}


Regarding the ActiTest results, 56.25\% of the patients ( 9 of them) have presented values corresponding to the A3 inflammation degree, $12.5 \%$ (2 of them) were regarded as A2, while $25 \%$ (4 of them) were borderline between $\mathrm{A} 1$ and $\mathrm{A} 2$ and $6.25 \%$ (one of them) was borderline between $\mathrm{A} 0$ and $\mathrm{A} 1$.

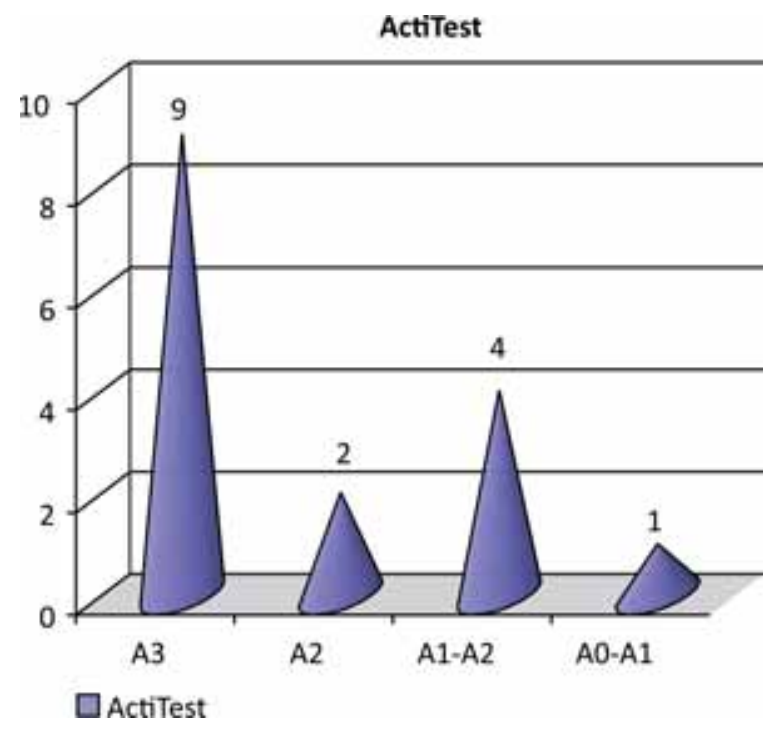

FIGURE 2. The ActiTests results

The SteatoTest's results were: 5 patients with S0 steatosis, 2 patients borderline between $\mathrm{S} 0$ and $\mathrm{S} 1$, 4 patients borderline between $\mathrm{S} 1$ and S2, 2 patients with $\mathrm{S} 1$ steatosis, 2 patients with $\mathrm{S} 2$ steatosis and only one patient with advanced S3 steatosis.

The NashTest's scores indicate a majority (7patients) with 0 degree (no inflammation), 5 patients with N2 and 4 patiens with N1 (possible NASH).

Regarding the AshTest's results, an overwhelming majority of 15 patients scored 0 - no alcoholic induced hepatic inflammation and only one patient scored $\mathrm{H} 1$ - a moderate alcohol induced hepatic inflammation.

Moreover, during this study we calculated the " $r$ " value of the linear correlation between the values of FibroTest and those of FibroScan $(\mathrm{r}=0.247)$, between the values of ActiTest and FibroScan $(\mathrm{r}=0.378)$, between the ones obtained at the SteatoTest and FibroScan $(r=0.013)$, between the ones of NashTest and FibroScan $(\mathrm{r}=0.146)$ and also between the values of AshTest and FibroScan ( $\mathrm{r}=0.108)$.

During the analysis of the obtained data, we observed a correlation between the fibrosis degree $(\mathrm{F})$ and the values of the same F obtained by FibroScan (we shall remind that for an F0-F1 the values should stay below $7.1 \mathrm{kPa}, \mathrm{F} 2$ is between 7.1 and $9.5 \mathrm{kPa}$,
F3 between 9.5 and $13 \mathrm{kPa}$ and $\mathrm{F} 4$ should exceed $13 \mathrm{kPa}$ ). Thus, in 11 of the 16 studied patients, the numbers obtained by FibroScan correspond to the degree of fibrosis calculated by FibroMax.

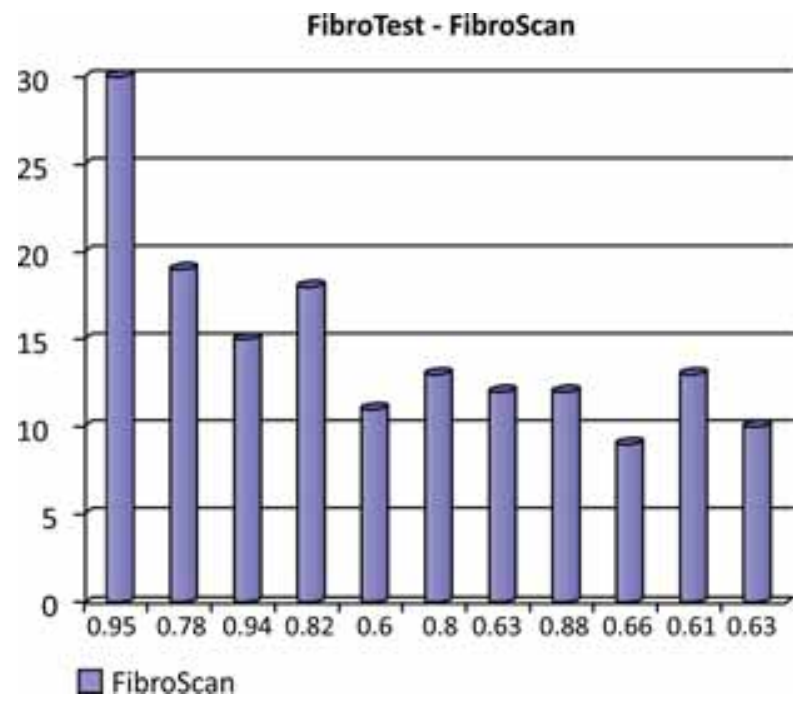

FIGURE 3. Correlations between FibroScan and FibroTest

\section{CONCLUSIONS}

Chronic hepatitis $\mathrm{C}$ contributes to $25 \%$ and even up to $40 \%$ of all chronic hepatic diseases and represents the cause of $40 \%$ of all the hepatic transplants (6).

The patients suffering from chronic hepatitis $\mathrm{C}$ are usually diagnosed in their thirties to fifties, when they can already have different degrees of hepatic fibrosis (from moderate to advanced) (6).

The methods used for the quantification of the heaptic fibrosis degree have had a tendency over the years to replace the invasive ones(like punch biopsy) with the non-invasive, ultramodern, such as the testing of biological markers in the FibroMax investigation and also the FibroScan device (5).

In this study we analysed a batch of 16 patients diagnosed with chronic hepatitis $\mathrm{C}$ and tested by both means, with different degrees of fibrosis (from $\mathrm{F} 2$ to $\mathrm{F} 4)$ and of virus induced inflammation (A0-A1-A3) and FibroScan results between $7.8 \mathrm{kPa}$ and $66.4 \mathrm{kPa}$.

By calculating the correlations of the values obtained by the two means (the "r" values), we established that the two methods are not necessarily comparable, but doing both of them is necessary for an accurate evaluation of the hepatic fibrosis and also in case of including the patient in a chronic hepatitis treatment programme. 


\section{REFERENCES}

1. http://www.cdt-babes.ro/articole/hepatita c infectie.php

2. Morra R, Munteanu M, Imbert-Bismut F, Messous D, Ratziu V, Poynard T. FibroMAX: Towards a new universal biomarker of liver disease? Expert Review of Molecular Diagnostics, 2007 Sep;7(5):481-90. https://www.ncbi.nlm.nih.gov/pubmed/17892356

3. http://www.cdt-babes.ro/noutati/fibroscan.php

4. http://www.mymed.ro/cum-se-interpreteaza-rezultatul-fibroscan.html

5. http://www.cdt-babes.ro/articole/evaluare-fibroza-hepatica.php

6. Gerald L. Mandell, John E. Bennett, Raphael Dolin. Principles and Practice of Infectious Disease - 7th edition, 2010 pg 2165

7. Gudowska M, Wojtowicz E, Cylwik B, Gruszewska E, Chrostek L. The Distribution of Liver Steatosis, Fibrosis, Steatohepatitis and
Inflammation Activity in Alcoholics According to FibroMax Test. Advances in Clinical and Experimental Medicine. 2015 Sep-Oct; 24(5):823- 7. doi:10.17219/acem/28485. https://www.ncbi.nlm.nih. gov/pubmed/26768633

8. Fouad A, Sabry D, Ahmed R, Kamal M, Allah SA, Marzouk S, Amin M, Abd El Aziz R, El Badri A, Khattab H, Helmy D. Comparative diagnostic study of biomarkers using FibroMax ${ }^{\mathrm{TM}}$ and pathology for prediction of liver steatosis in patients with chronic hepatitis $C$ virus infection: an Egyptian study. International Journal of General Medicine 2013 Mar 12;6:127-34. doi: 10.2147/IJGM.S36433. Print 2013, https://www.ncbi.nlm.nih.gov/pubmed/23662075 